\title{
The Spectral Geometry of Flat Disks
}

\section{Citation}

Brooks, Robert, Yakov Eliashberg, and Curtis T. McMullen. 1990. The spectral geometry of flat disks. Duke Mathematical Journal 61(1): 119-132.

\section{Published Version}

doi:10.1215/S0012-7094-90-06106-X

\section{Permanent link}

http://nrs.harvard.edu/urn-3:HUL.InstRepos:3446033

\section{Terms of Use}

This article was downloaded from Harvard University's DASH repository, and is made available under the terms and conditions applicable to Other Posted Material, as set forth at http:// nrs.harvard.edu/urn-3:HUL.InstRepos:dash.current.terms-of-use\#LAA

\section{Share Your Story}

The Harvard community has made this article openly available.

Please share how this access benefits you. Submit a story.

Accessibility 


\section{THE SPECTRAL GEOMETRY OF FLAT DISKS \\ ROBERT BROOKS, YAKOV ELIASHBERG AND C. MCMULLEN}

In [6], Mark Kac raised the question of whether two domains in the Euclidean plane $\mathbb{R}^{2}$ which have the same spectrum of the Laplacian are congruent. Central to his approach to this question was the fact that certain invariants of the Laplacian for such a domain $D$, called the heat invariants, are expressible in terms of the area of $D$ and integrals over the boundary $\partial D$ of the domain of terms involving the length, the geodesic curvature, and derivatives of these quantities-see $\$ 1$ below for a discussion.

One is naturally led by this approach to consider the extent to which the geometry of $D$ in a neighborhood of $\partial D$ governs the spectrum of $D$. To that end, let us say that two flat disks $D_{1}$ and $D_{2}$ are isometric near the boundary if there are neighborhoods of $\partial D_{1}$ and $\partial D_{2}$ which are isometric. Furthermore, we will say that $D_{1}$ and $D_{2}$ are piecewise isometric near the boundary if there are neighborhoods $N_{1}$ and $N_{2}$ of $\partial D_{1}$ and $\partial D_{2}$ such that $N_{1}$ can be subdivided into finitely many pieces and rearranged to obtain $N_{2}$.

It is easy to see that if $D_{1}$ and $D_{2}$ are piecewise isometric near the boundary and have the same area, then all of the heat invariants of $D_{1}$ and $D_{2}$, and indeed all such integrals over the boundary, must agree. It is also easy to see that if $D_{1}$ and $D_{2}$ are planar disks which are isometric near the boundary, then $D_{1}$ and $D_{2}$ are congruent.

However, it is an interesting fact that there are flat disks $D_{1}$ and $D_{2}$ which are isometric near the boundary, but which are not themselves isometric. Such disks immerse into the plane so that they have a common boundary curve. We will see how to construct such examples in $\$ 2$ below.

In $\S 3$ and $\S 4$ below, we will then show:

THEOREM 1. There are compact flat disks $D_{1}$ and $D_{2}$ which are isometric near the boundary, but which are not isospectral for either Dirichlet or Neumann boundary conditions.

Using similar techniques, we will also show:

THEOREM 2. There exist planar disks $D_{1}$ and $D_{2}$ which are piecewise isometric near the boundary, but which are not isospectral for either Dirichlet or Neumann boundary conditions.

Finally, in $\S 5$ we will establish the analogue of Theorem 1 in dimensions greater than 2 .

Received August 12, 1989. 
It is a pleasure to thank the Mathematical Sciences Research Institute for its support during the development of this work.

1. The Heat Invariants. Let $D$ be a compact, connected Riemannian manifold, with boundary $\partial D$. A choice of self-adjoint boundary conditions (for our purposes, either Dirichlet or Neumann conditions) turns the Laplacian $\Delta$ into a positive semi-definite operator, with discrete spectrum tending to $+\infty$. We denote the spectrum by

$$
0=\lambda_{0}^{N}(D)<\lambda_{1}^{N}(D) \leqslant \lambda_{2}^{N}(D) \leqslant \cdots
$$

for Neumann conditions, and

$$
0=\lambda_{0}^{D}(D)<\lambda_{1}^{D}(D) \leqslant \lambda_{2}^{D}(D) \leqslant \cdots
$$

for Dirichlet conditions.

The Heat Kernel $H_{t}^{N}(x, y)\left(\operatorname{resp} . H_{t}^{D}(x, y)\right)$ is defined by

$$
\begin{aligned}
& H_{t}^{N}(x, y)=\sum_{i=0}^{\infty} e^{-\lambda_{i} t} \phi_{i}^{N}(x) \phi_{i}^{N}(y) \\
& H_{t}^{D}(x, y)=\sum_{i=0}^{\infty} e^{-\lambda_{i} t} \phi_{i}^{D}(x) \phi_{i}^{D}(y)
\end{aligned}
$$

where $\phi_{i}^{N}$ (resp. $\phi_{i}^{D}$ ) runs over an orthonormal basis of eigenfunctions with eigenvalue $\lambda_{1}$.

The trace of the Heat Kernel is defined by

$$
\begin{aligned}
& \operatorname{tr}\left(H_{t}^{N}\right)=\int_{D} H_{t}^{N}(x, x) d x \\
& \operatorname{tr}\left(H_{t}^{D}\right)=\int_{D} H_{t}^{D}(x, x) d x
\end{aligned}
$$

and has a well-known asymptotic expansion as $t \rightarrow 0$ given by

$$
\begin{aligned}
& \operatorname{tr}\left(H_{t}^{N}\right) \sim \frac{1}{(4 \pi t)^{n / 2}}\left(\sum_{i=0}^{\infty}\left(a_{i / 2}^{N}\right) t^{i / 2}\right) \\
& \operatorname{tr}\left(H_{t}^{D}\right) \sim \frac{1}{(4 \pi t)^{n / 2}}\left(\sum_{i=0}^{\infty}\left(a_{i / 2}^{D}\right) t^{i / 2}\right)
\end{aligned}
$$

where the $a_{i}^{N}$ (resp. $a_{i}^{D}$ ) are given by integrating local expressions in the curvature over $D$ and local expressions in the geodesic curvature over $\partial D$, and $n=\operatorname{dim}(D)$. 
The case of Dirichlet boundary conditions has been studied extensively, but there is little difference of an analytic nature between the two cases-see [3] for a general reference. In what follows, if we do not specify Dirichlet or Neumann boundary conditions, it will be understood that Dirichlet conditions are intended, but that an analogous formula would be possible using Neumann conditions.

In the case when $D$ is flat, all the curvature terms disappear, so that we have, when $\operatorname{dim}(D)=2$,

$$
\begin{aligned}
a_{0}(D) & =\operatorname{area}(D) \\
a_{1 / 2}(D) & =-\frac{1}{\sqrt{8} \pi} \text { length }(\partial D) \\
a_{1}(D) & =\frac{\chi(D)}{6}
\end{aligned}
$$

where $\chi(D)$ is the Euler characteristic of $D$ (note that this last is an integral over $\partial D$, by the Gauss-Bonnet Theorem with boundary).

The $a_{i}$ 's are called the heat invariants of $D$, and are visibly spectral invariants of $D$. It is evident that if $D_{1}$ and $D_{2}$ have the same area and are isometric near the boundary, or even piecewise isometric near the boundary, then all of the $a_{i}$ 's agree for $D_{1}$ and $D_{2}$, since they are expressed by integrals which agree piecewise.

One might be tempted to believe that the $a_{i}$ 's determine a lot of the geometry of $D$. Indeed, this temptation seems to have motivated Kac in [6]. That this is partially true was demonstrated by Melrose in [8], who showed that when $D$ is a plane domain, the $a_{i}^{D}$ 's determine $\partial D$ (and hence $D$ ) up to a compact set of possibilities. However, this compact set may include certain degeneracies in $\partial D$, as in the following picture:

It is clear that one may pinch the middle of this region so as to keep the $a_{i}$ 's bounded. However, the region $D$ tends in the limit to two disks joined at a point.

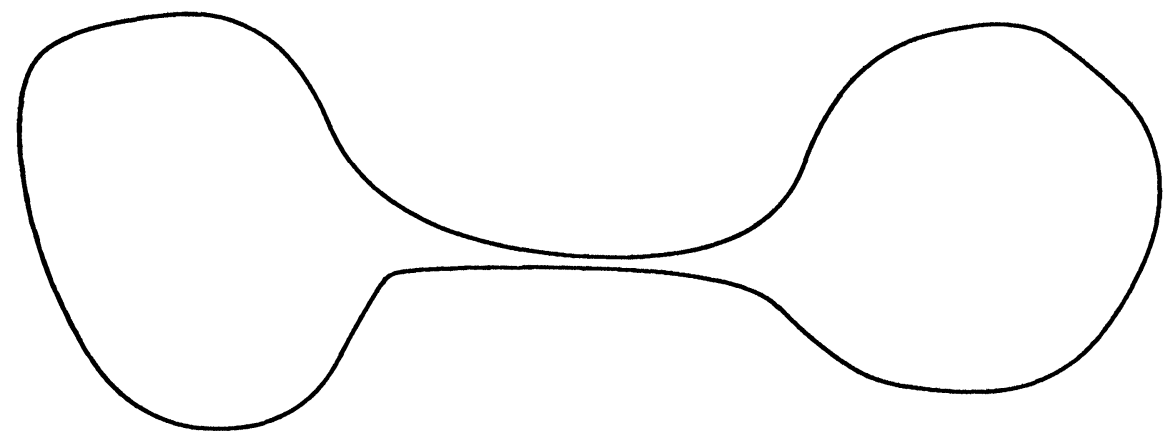

Figure 1 
The question was then raised as to whether this pinching could be detected spectrally.

See [11] for a discussion of this question. In particular, it is shown there and in [9] how the determinant of the Laplacian serves as a non-local spectral invariant which detects this kind of degeneracy. We will return to this line of thought in $\S 4$ below.

2. Flat Disks. Let $D$ be a compact flat two-dimensional manifold with boundary $\partial D$. Then there is an isometric immersion $h: \widetilde{D} \rightarrow \mathbb{R}^{2}$ of the universal cover $\tilde{D}$ of $D$ into the Euclidean plane, which is unique up to Euclidean motion, and is described as follows: given a point $x \in \tilde{D}$, there is an isometry between a neighborhood of $x$ and a neighborhood of a point in $\mathbb{R}^{2}$. This isometry can then be extended uniquely along all paths in $\tilde{D}$ to give $h$.

When $D$ is a disk, then $\widetilde{D}=D$, and so $h$ gives an immersion of $D$ into $\mathbb{R}^{2}$. The restriction of $h$ to $\partial D$ is then a closed curve $\gamma$ in the plane.

If $\gamma$ has no self-intersection, then the Jordan Curve Theorem says that $\gamma$ bounds a unique disk, which must then be $D$, and we have exhibited $D$ as a plane domain. However, if $\gamma$ has self-intersections, then the situation is more complicated, and in particular there could conceivably be more than one immersed disk $D$ which has $\gamma$ as a boundary.

Such a curve was studied by Blank [1], who attributed the example to Milnor (see [7] for a historical discussion), and independently by Eliashberg [5], and is shown below in Figure 2:

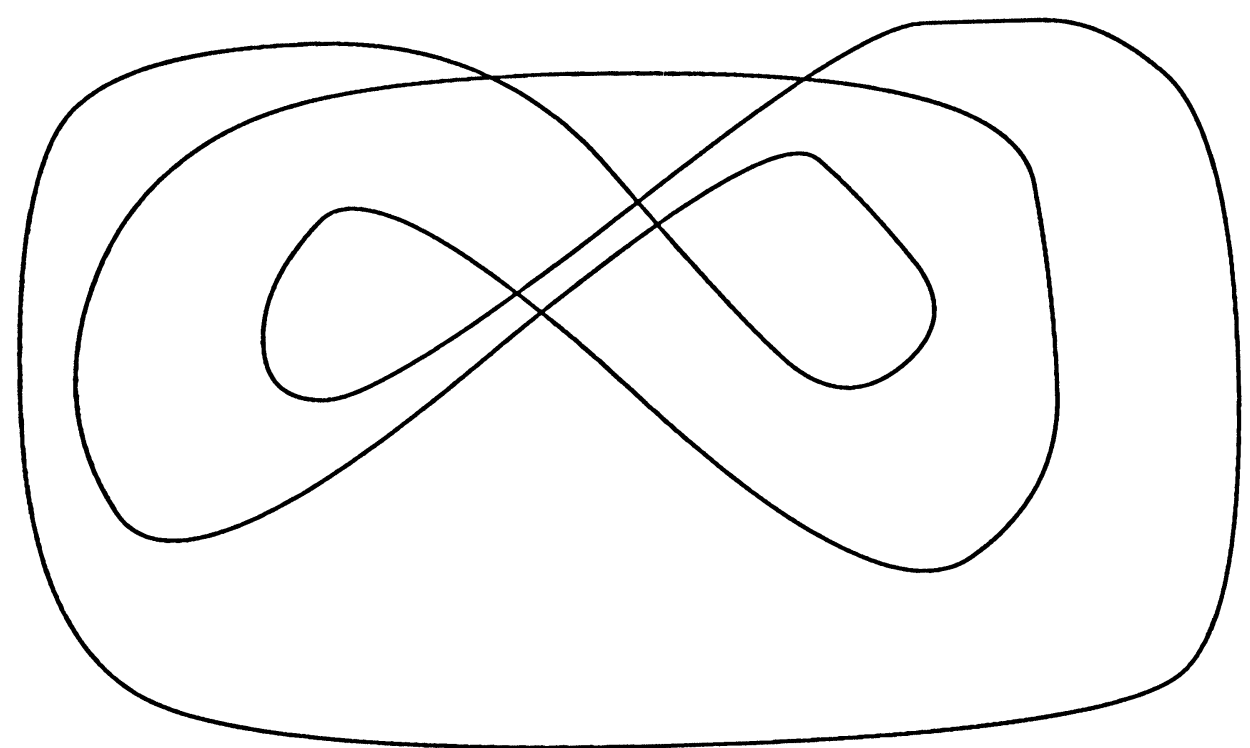

FiGURE 2 


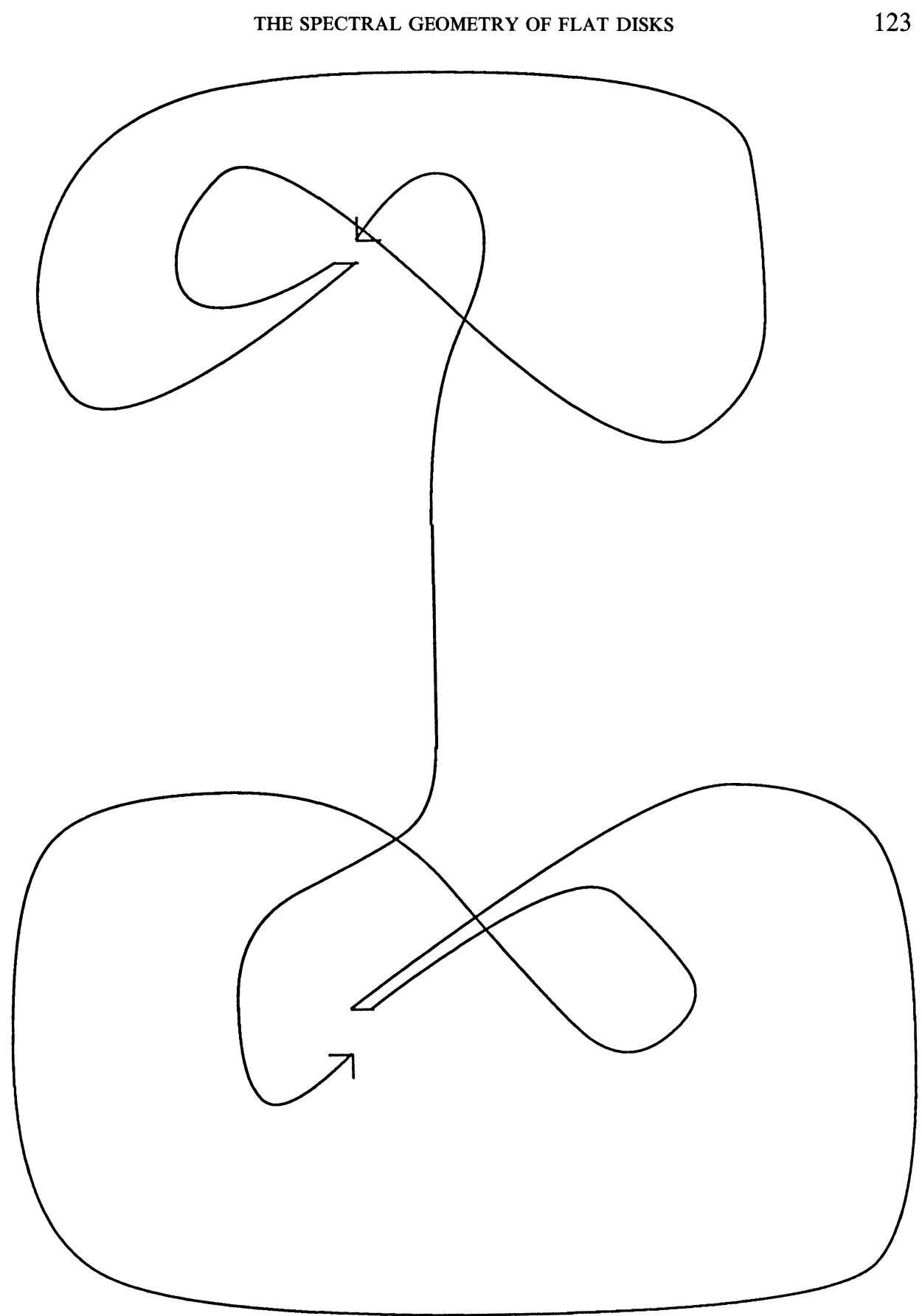

FIGURE 3: The Disk $D_{1}$ 
124

BROOKS, ELIASHBERG, AND MCMULLEN

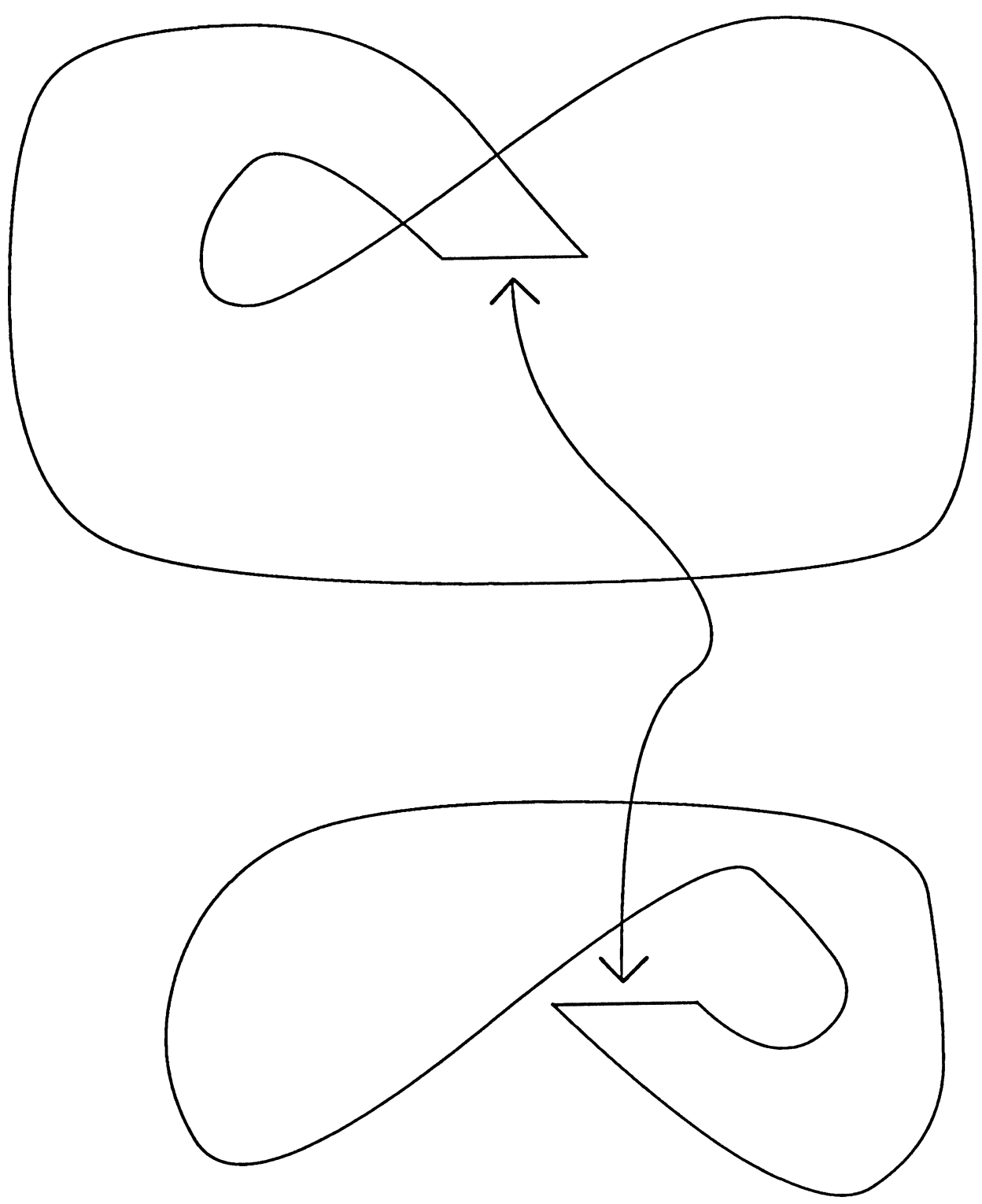

Figure 4: The Disk $D_{2}$ 
It is not difficult to see the two disks $D_{1}$ and $D_{2}$ bounded by this curve. We illustrate this in Figures 3 and 4 below, where, for ease in visualization, we have cut the disk $D_{1}$ (resp. $D_{2}$ ) into two pieces to reduce the overlapping.

Notice that $D_{1}$ and $D_{2}$ are isometric near their boundaries-indeed, a neighborhood of $\partial D_{1}$ in $D_{1}$ (resp. $\partial D_{2}$ in $D_{2}$ ) is given by a one-sided neighborhood of $\gamma$.

3. Neumann Boundary Conditions. In this section, we show that, for appropriate choices of $\gamma, D_{1}$ and $D_{2}$ are not isospectral for Neumann boundary conditions.

Our main tool is:

Cheeger's ineQuality (Neumann CaSe) [4]. For D a surface with boundary,

$$
\lambda_{1}^{N}(D) \geqslant \frac{1}{4} h^{2}
$$

where

$$
h=\inf _{L} \frac{\text { length }(L)}{\min (\operatorname{area}(A), \operatorname{area}(B))},
$$

as $L$ runs over all curves dividing $D$ into two pieces $A$ and $B$.

Cheeger's inequality is, of course, valid for all dimensions, but for simplicity in notation we will restrict to the two-dimensional case.

We now choose $\gamma$ so that $D_{1}$ has a long, narrow tube at the place which is cut open in Figure 3, but $D_{2}$ has no such narrow tube.

To estimate $\lambda_{1}^{N}\left(D_{1}\right)$, we consider a test function $f_{\gamma}$ which is $\equiv c_{1}$ on one lobe in Figure $3, \equiv-c_{2}$ on the other lobe, and which changes linearly along the narrow tube, where $c_{1}$ and $c_{2}$ are two positive constants chosen so that $\int_{D_{1}} f_{\gamma}=0$. One choice is to let $c_{2}$ be approximately the area of the first lobe, and $c_{1}$ be approximately the area of the second lobe.

We now compute the Rayleigh quotient

$$
\frac{\int\left\|\operatorname{grad}\left(f_{\gamma}\right)\right\|^{2}}{\int\left\|f_{\gamma}\right\|^{2}}
$$

But $\left\|\operatorname{grad}\left(f_{\gamma}\right)\right\|$ is supported only on the tube, and its value depends only on the length of the tube, not its width. It follows that as the width of the tube tends to 0 , the Rayleigh quotient, and hence $\lambda_{1}^{N}\left(D_{1}\right)$, tends to 0 as well.

On $D_{2}$, however, there is no such narrow tube, and in the limit, $D_{2}$ converges to a smooth disk. It follows that $h\left(D_{2}\right)$ is bounded away from zero, and hence, by Cheeger's inequality, the same is true for $\lambda_{1}^{N}\left(D_{1}\right)$. It follows that, as the tube narrows, $\lambda_{1}^{N}\left(D_{1}\right)$ is strictly less than $\lambda_{1}^{N}\left(D_{2}\right)$. 
4. Dirichlet Boundary Conditions. In this section, we will show how to choose $\gamma$ so that $D_{1}$ and $D_{2}$ are not isospectral for Dirichlet boundary conditions.

We first remark that Cheeger's inequality is also available in this case:

Cheeger's inequality (Dirichlet CASE) [4].

$$
\lambda_{0}^{D}(D) \geqslant \frac{1}{4} h^{2},
$$

where

$$
h=\inf _{L} \frac{\text { length }(L)}{\operatorname{area}(\operatorname{int}(L))},
$$

as $L$ runs over closed curves in $D$ dividing $D$ into two components, one of which does not contain $\partial D$. We call this component int $(L)$.

It is possible to analyze the curve $L$ which realizes this minimum-see [2] for a discussion-and from this arrange $\gamma$ so that $\lambda_{0}^{D}\left(D_{1}\right)>\lambda_{0}^{D}\left(D_{2}\right)$. The following variant of Cheeger's inequality, due to Osserman [10], is much easier to work with:

THEOREM (OSSERMANN) [10]. If $D$ is a compact flat disk, then

$$
\lambda_{0}^{D}(D) \geqslant \frac{1}{4 \rho^{2}}
$$

where $\rho$ is the radius of the largest disk contained in $D$.

Note that Osserman's theorem is usually stated for planar disks, but was proved in [10] in the more general case considered here, and indeed under the more general assumption that $D$ is simply connected and has non-positive curvature.

Note also the upper bound

$$
\lambda_{0}^{D}(D) \leqslant \frac{(\text { const })}{\rho^{2}}
$$

where (const) is the lowest Dirichlt eigenvalue of the unit disk, as can be seen from the fact that $\lambda_{0}^{D}$ is strictly decreasing under inclusion of domain.

We may now choose our curve $\gamma$ in the following way, as shown in Figure 5, which shows the right-hand lobe of $\gamma$ : the outside curve becomes a large lobe, while the inner curves wiggle in and out.

In Figures 6 and 7, we show the corresponding parts of the disks $D_{1}$ and $D_{2}$. Note that $D_{2}$ has a very large right-hand lobe, and hence $\lambda_{0}^{D}\left(D_{2}\right)$ is very small. However, the corresponding parts of $D_{1}$ contain no balls of large radius (they are destroyed by the wiggling). It follows from Osserman's theorem that $\lambda_{0}^{D}\left(D_{1}\right)$ is large, and in particular $\lambda_{0}^{D}\left(D_{1}\right)>\lambda_{0}^{D}\left(D_{2}\right)$. 


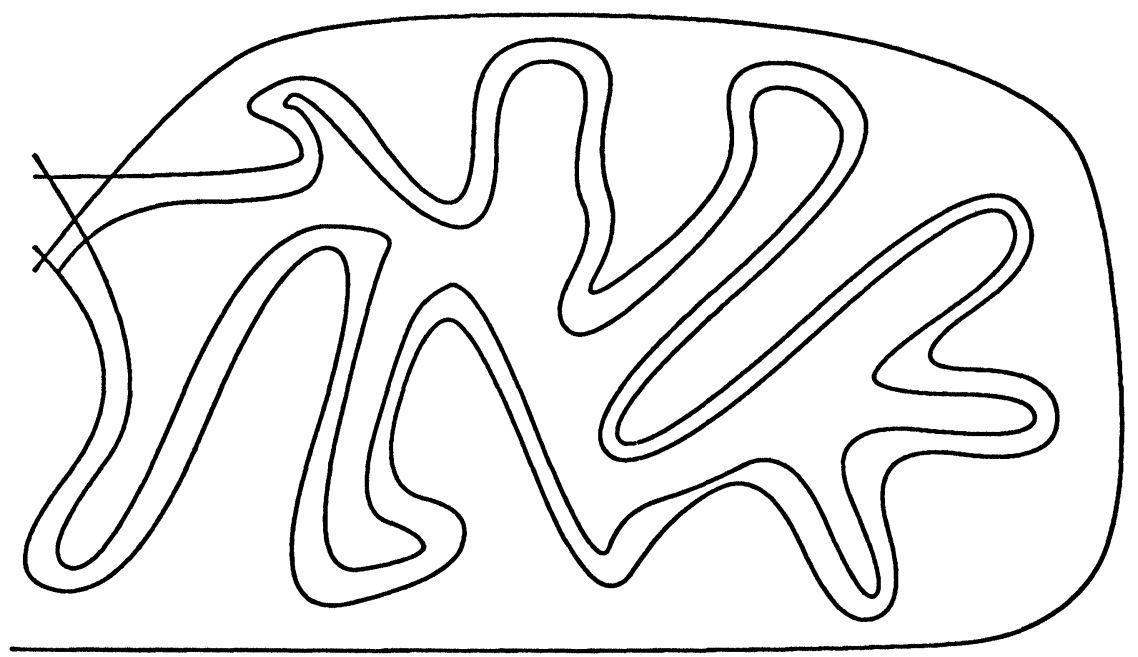

Figure 5

Note that this wiggling can be done independent of the narrowing of the tube of $\S 3$. In particular, this completes the proof of Theorem 1.

Another, less elementary, approach is given by the recent theorem of Osgood, Phillips, and Sarnak [9]. Their theorem is stated for plane domains, but at the first step they pass to flat matrics on the unit disk via the Uniformization Theorem, so their proof is valid without change to the category of compact flat disks. They show that as the boundary of a domain pinches in on itself so that the heat invariants remain bounded, then $-\log (\operatorname{det}(\Delta))$ must tend to $+\infty$, where $\operatorname{det}(\Delta)$ is the determinant of the Laplacian for Dirichlet boundary conditions, and is a spectral invariant.

From this we conclude that, in the construction we gave for Neumann boundary conditions in $\S 3$, as the tube narrows on $D_{1}, D_{1}$ and $D_{2}$ must differ spectrally for the Dirichlet as well as Neumann conditions, since $-\log (\operatorname{det}(\Delta))$ remains bounded for $D_{2}$. In this approach, though, one is hard put to decide which eigenvalues for the Dirichlet conditions differ.

We now turn to Theorem 2. We observe that the planar disks shown in Figure 8 below are evidently of the same area and piecewise isometric near the boundary. However, the disk on the right has a thin neck in the middle, while the one on the left does not, so they differ for Neumann boundary conditions. Furthermore, the left disk has a large embedded ball while the right one does not, so they differ also for Dirichlet conditions.

5. Higher Dimensions. In this section, we will show how the construction of $\S 2$ generalizes to give flat $n$-dimensional disks $D_{1}^{n}$ and $D_{2}^{n}$ which are isometric near their boundaries but are not isospectral. 

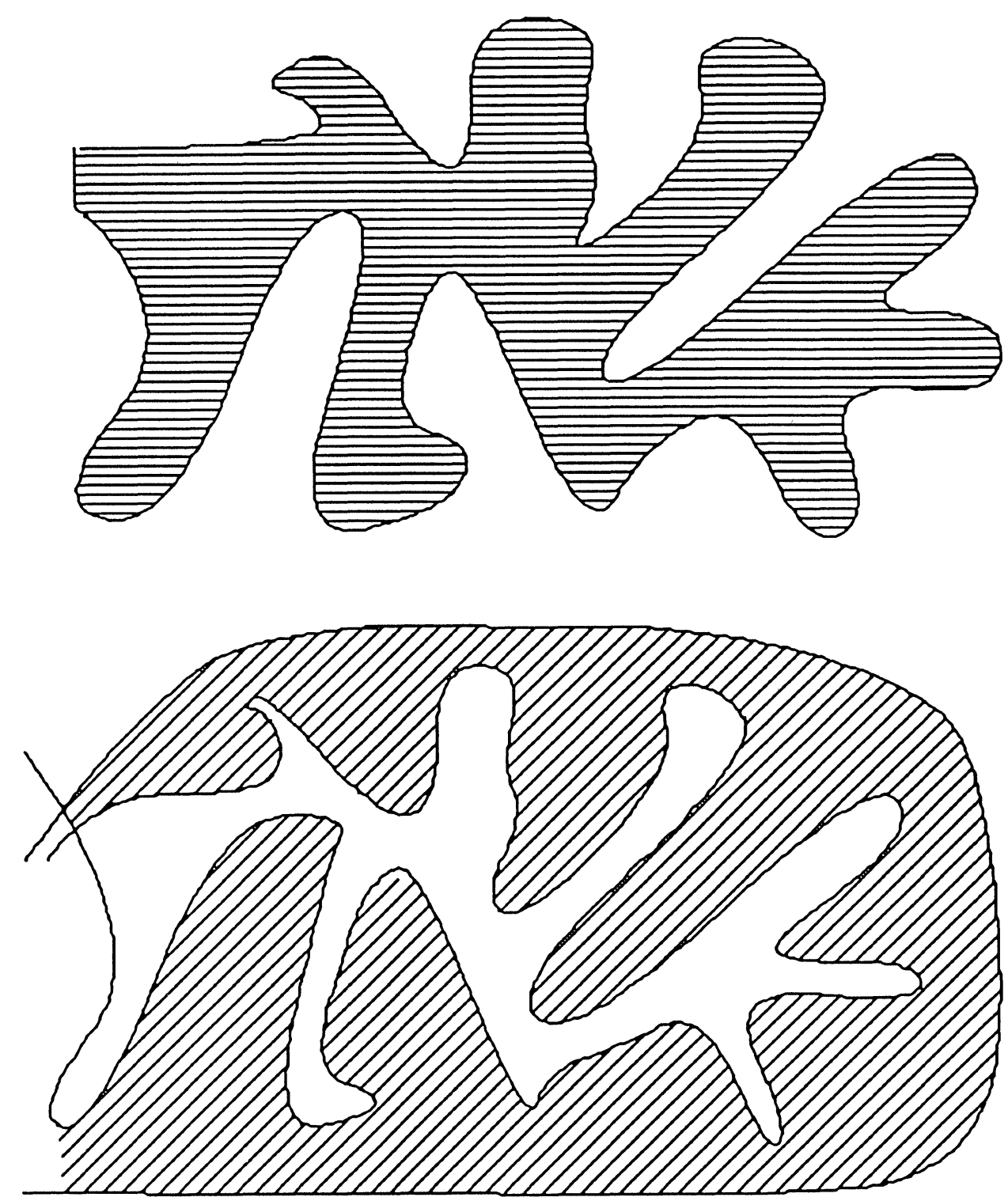

FIGURE 6: The wiggled $D_{1}$ 

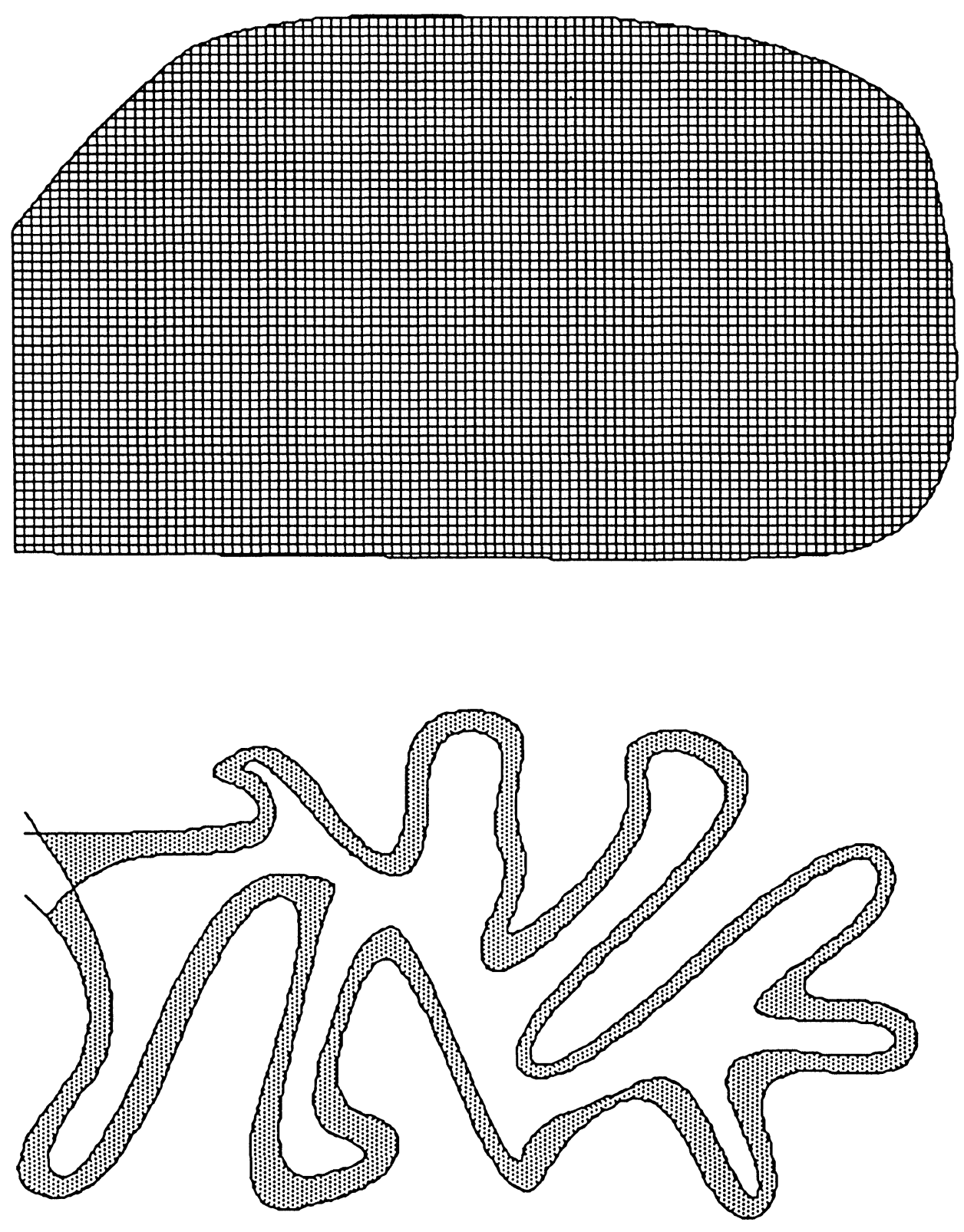

FIGURE 7: The wiggled $D_{2}$ 


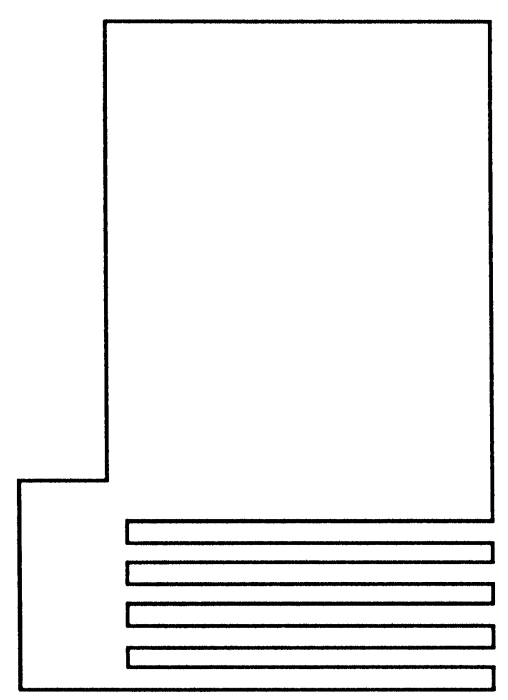

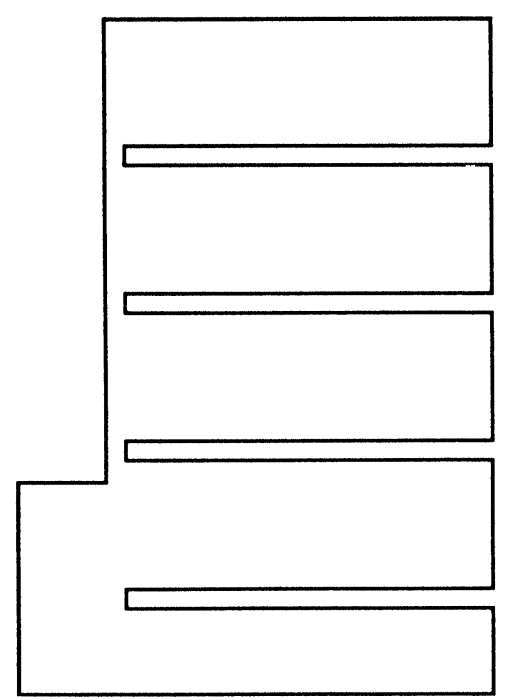

Figure 8

We begin with the following observation: let $U$ be a region in $\mathbb{R}^{2}$ which is symmetric about the $x_{1}$-axis. Let us denote by $U^{n}$ the region in $\mathbb{R}^{n}$ obtained from $U$ by including $\mathbb{R}^{2}$ in $\mathbb{R}^{n}$, and rotating $U$ by the orthogonal group $S O(n-1)$ keeping the $x_{1}$-axis fixed. It is then obvious that if $U$ is topologically a disk, then $U^{n}$ is topologically an $n$-dimensional disk, and its boundary is an $n$-1-dimensional sphere.

To construct $D_{1}^{n}$ and $D_{2}^{n}$, we now proceed as follows: letting $D$ be the standard disk in $\mathbb{R}^{2}$, we let $U_{1}$ be obtained by joining $D$ with two copies of the disk $D_{1}$ of Theorem 1 placed symmetrically about the $x_{1}$-axis, and let $U_{2}$ be obtained analogously from $D_{2}$. Then the corresponding $n$-disks $U_{1}^{n}$ and $U_{2}^{n}$ are the desired disks $D_{1}^{n}$ and $D_{2}^{n}$. Their common boundary is the $(n-1)$-sphere $S$ which is the $S O(n-1)$ orbit of $\partial U_{1}=\partial U_{2}$. Note that the intersection of $D_{1}^{n}$ with any plane containing the $x_{1}$-axis is isometric to $U_{1}$, and the intersection of $D_{2}^{n}$ with any plane containing the $x_{1}$-axis is isometric to $U_{2}$.

We now claim that $D_{1}^{n}$ and $D_{2}^{n}$ are spectrally distinct.

This is clear when we look at Neumann boundary conditions, for the same reasons as in $\S 3$. $D_{1}^{n}$ can be cut into two pieces along the thin neck by a hypersurface of the form $\mathbb{D}^{1} \times S^{n-2}$ and has small area, but $D_{2}^{n}$ cannot be so divided.

Dirichlet conditions look more problematic, since both Osserman's theorem and the theorem of Osgood-Phillips-Sarnak are strictly two-dimensional arguments. 
However, we recall the standard fact that $\lambda_{0}^{D}$ is always a simple eigenvalue. It follows that the corresponding eigenfunction $\phi_{0}$ is invariant under the natural $S O(n-1)$-action, since the same is true of $D_{1}^{n}$ and $D_{2}^{n}$ by construction. It follows that, for any point $x, \operatorname{grad}\left(\phi_{0}\right)$ always points in direction contained in the plane containing $x$ and the $x_{1}$-axis. Therefore, the Rayleigh quotient of $\phi_{0}$ is given by the Rayleight quotient of $\phi_{0}$ restricted to any plane containing the $x_{1}$-axis. This is then estimated by Osserman's theorem, exactly as in $\S 4$.

\section{REFERENCES}

1. S. J. Blank, Extending immersions of the circle, Ph.D. thesis, Brandeis University, 1967.

2. R. Brooks AND P. WaKsman, The first eigenvalue of a scalene triangle, Proc. AMS 100 (1987), pp. $175-182$.

3. I. Chavel, Eigenvalues in Riemannian Geometry, Academic Press, 1984.

4. J. Cheeger, "A lower bound for the smallest eigenvalue of the Laplacian," in Gunning, Problems in Analysis, (1970), pp. 195-199.

5. Y. ElIASHBERG, On singularities of folding type, Izvestia AN SSSR, ser. mat., 34 (1970), pp. $1111-1127$.

6. M. KAC, Can one hear the shape of a drum?, Amer. Math. Month. 73 (1966), pp. 1-23.

7. M. L. MARX, Extensions of Normal Immersions of $S^{1}$ into $\mathbb{R}^{2}$, Trans. AMS 187 (1974), pp. 309-326.

8. R. MELrose, Isospectral sets of drumheads are compact in $C^{\infty}$, MSRI preprint, 1984.

9. B. Osgood, R. Phillips, AND P. SARnaK, Compact isospectral sets of metrics, J. Funct. Anal. 80 (1988) pp. 212-234.

10. R. OSSERman, A note on Hayman's theorem on the bass note of a drum, Comm. Math. Helv. 52 (1977). pp. 545-555.

11. P. SARnAK, "Determinants of Laplacians heights and finiteness," P. Rabinowitz and E. Zehnder, Analysis, Etc., Academic Press 1990, pp. 601-622.

Brooks: Department of Mathematics, University of Southern California, Los Angeles, CALIFORNIA 90089-1113.

Eliashberg: Department of Mathematics, Stanford University, Stanford, California 943052125.

McMullen: Department of Mathematics, Princeton University, Princeton, New Jersey 08544 . 